\title{
ОЦЕНКА ДИАСТОЛИЧЕСКОЙ ФУНКЦИИ МИОКАРДА У ПАЦИЕНТОВ С БОЛЕВЫМ СИНДРОМОМ В ГРУДНОЙ КЛЕТКЕ И АНГИОГРАФИЧЕСКИ НЕИЗМЕННЫМИ КОРОНАРНЫМИ АРТЕРИЯМИ МЕТОДОМ СИНХРОНИЗИРОВАННОЙ С ЭКГ ОДНОФОТОННОЙ ЭМИССИОННОЙ КОМПЬЮТЕРНОЙ ТОМОГРАФИИ
}

Э. А. Хачирова ${ }^{1 凶}$, Л. Е. Самойленко², О. П. Шевченко

${ }^{1}$ Российский национальный исследовательский университет имени Н. И. Пирогова, Москва, Россия

2 Российская медицинская академия постдипломного образования, Москва, Россия

На протяжении многих лет остается актуальной тема диагностики и тактики ведения пациентов с ангиографически неизмененными или малоизмененными коронарными артериями (КА). Целью исследования было оценить диастолическую функцию у пациентов с болевым синдромом в грудной клетке и неизмененными/малоизмененными КА (H/MKA) по данным синхронизированной с ЭКГ однофотонной эмиссионной компьютерной томограсии (С-ОЭКТ/КТ). В исследование вошли 49 пациентов с болевым синдромом в области грудной клетки, положительными результатами нагрузочного теста и Н/MКА по данным коронароангиографии (КАГ). Всем пациентам выполнили С-ОЭКТ/КТ миокарда по двухдневному протоколу. После исследования пациенты были разделены на три группы: 17 пациентов с микрососудистой стенокардией составили группу 1; 22 пациента с пограничной артериальной гипертензией (АГ), или гипертонической болезнью (ГБ) І стадии, ассоциирующейся с вторичной микрососудистой дисфункцией - группу 2. Группу контроля, или группу 3, составили 10 практически здоровых лиц, у которых при КАГ были исключены заболевания со стороны сердечно-сосудистой системы, сопровождающиеся изменениями коронарных артерий и нарушениями перфузии миокарда при проведении ОЭКТ. У большинства пациентов в группах 1 и 2 были выявлены нарушения диастолической функции, при этом в группе 2 они глубже и усугубляются при нагрузке. Наиболее чувствительный параметр диастолической функции - средняя скорость наполнения ЛЖ в первую треть диастолы (MFR/3) - изменен практически у всех пациентов групп 1 и 2. У пациентов группы контроля изменения не выявлены. Таким образом, у пациентов с болью в груди, положительным стресс-тестом и Н МКА выявлены признаки диастолической дисфункции ЛЖ (ДДФ) и дальнейшее ее ухудшение при нагрузке. Эти пациенты могут представлять собой группу риска развития сердечной недостаточности с сохраненной фракцией выброса.

Ключевые слова: однофотонная эмиссионная компьютерная томография, ангиографически неизмененные/малоизмененные эпикардиальные коронарные сосуды, диастолическая функция, перфузионная сцинтиграфия, микрососудистая стенокардия

Благодарности: авторы благодарят Виктора Константиновича Сычева за возможность воспользоваться радиоизотопной лабораторией и оборудованием в процессе сбора материала.

Информация о вкладе авторов: Э. А. Хачирова подбирала пациентов, оформляла документацию для проведения исследования, участвовала в проведении велоэргометрии и сцинтиграфического исследования, обрабатывала полученные данные; Л. Е. Самойленко контролировала протокол исследования, проверяла полученные результаты; О. П. Шевченко контролировал соблюдение критериев включения в исследование и критериев исключения при отборе пациентов.

Соблюдение этических стандартов: исследование одобрено этическим комитетом РНИМУ им. Н. И. Пирогова (протокол № 150 от 15 декабря 2015 г.) Все пациенты подписали добровольное информированное согласие на проведение исследования.

Для корреспонденции: Эльвира Азреталиевна Хачирова

ул. Островитянова, д. 1, г. Москва, 117997; elchik09@mail.ru

Статья получена: 17.09.2018 Статья принята к печати: 11.11.2018 Опубликована онлайн: 23.02.2019

DOI: 10.24075 /vrgmu.2019.001

\section{ASSESSMENT OF DIASTOLIC FUNCTION IN PATIENTS WITH CHEST PAIN AND ANGIOGRAPHICALLY NORMAL CORONARY ARTERIES USING ECG-GATED SPECT}

Khachirova EA ${ }^{1} \otimes$, Samoylenko LE², Shevchenko OP ${ }^{1}$

${ }^{1}$ Pirogov Russian National Research Medical University, Moscow, Russia

${ }^{2}$ Russian Medical Academy of Postgraduate Education, Moscow, Russia

The diagnosis and treatment of patients with angiographically normal or near normal coronary arteries remains a clinically relevant problem. The aim of this study was to assess diastolic function in patients with chest pain and normal/near normal coronary arteries (NECA) using ECG-gated SPECT/CT. The study recruited 49 patients presenting with chest pain, a positive cardiac stress test and normal coronary arteries, as demonstrated by coronary angiography. All patients were ordered a myocardial SPECT/CT scan, which was performed according to a two-day protocol. After the scan, the patients were divided into 3 groups. Group 1 consisted of 17 patients with microvascular angina. Group 2 was composed of 22 patients with borderline-high blood pressure or stage I hypertensive heart disease associated with secondary microvascular dysfunction. Ten seemingly healthy individuals constituted the control group. According to coronary angiography, the controls had no cardiovascular pathologies accompanied by coronary artery disorders or impaired myocardial perfusion (SPECT). The majority of patients from groups 1 and 2 were found to have impaired diastolic function. The impairments were more pronounced in group 2 tended to exacerbate with stress. The most sensitive parameter of diastolic function, MFR/3, was outside the reference range in almost all patients in groups 1 and 2 . MFR/3 characterizes the mean filling rate of the left ventricle in the first third of diastole. The control group showed no symptoms of diastolic dysfunction. Thus, the patients with chest pain, a positive stress test and NECA had signs of left ventricular diastolic dysfunction exacerbated with stress. Such patients are at risk for heart failure with preserved ejection fraction. Keywords: single-photon emission computed tomography, microvascular angina, diastolic function, angiographically normal or near normal epicardial coronary arteries, myocardial perfusion

Acknowledgements: the authors wish to thank Viktor K. Sychev for kindly allowing them to use the equipment of the Laboratory for Radioisotopes

Author contribution: Khachirova EA recruited patients for the study, prepared the necessary documentation, participated in conducting the cardiac stress test and SPECT/CT, and processed the obtained data; Samoylenko LE controlled the course of the study and double-checked the obtained results; Shevchenko OP made sure inclusion and exclusion criteria were met.

Compliance with ethical standards: the study was approved by the Ethics Committee of Pirogov Russian Medical Research Medical University (Protocol 150 dated December 15, 2015). All patients gave informed consent to participate.

$\triangle$ Correspondence should be addressed: Elvira A. Khachirova Ostrovityanova 1, Moscow, 117997; elchik09@mail.ru

Received: 17.09.2018 Accepted: 11.11.2018 Published online: 23.02.2019

DOI: 10.24075/brsmu.2019.001 
C внедрением в повседневную клиническую практику эхокардиографии (ЭХОКГ) выяснилось, что сердечная недостаточность $(\mathrm{CH})$ с сохраненной систолической функцией (с фракцией выброса (ФВ) миокарда левого желудочка (ЛЖ) > 45\%) достаточно широко распространена во всем мире. Согласно данным ряда исследований (Фремингемское, 1948-2012 гг., Рочестерское эпидемиологическое, 1960-1984 гг. и др.), пациенты с сохраненной ФВЛЖ составляют от одной трети до половины больных с СН, и их общая численность неуклонно растет [1, 2]. Полагают, что одной из причин СН у таких пациентов является диастолическая дисфункция (ДДФ) [3-5]. При этом изолированная ДДФ встречается чаще у пациентов с Н/MKA [6].

Несмотря на определенные успехи, достигнутые в изучении ДДФ у больных с H/MKA, к настоящему времени вопросы этиологии и патогенеза нарушений процессов диастолы у этой категории пациентов окончательно не раскрыты [7, 8]. В литературе обсуждаются различные причины ДДФ и ассоциированной с ней $\mathrm{CH}$, в том числе гипертрофия миокарда ЛЖ, метаболические сдвиги, фиброз миокарда и коронарная микрососудистая дисфункция (МСД), которую многие авторы рассматривают не только в качестве основы ДДФ и СН, но еще и в качестве фактора риска неблагоприятных сердечно-сосудистых событий $[9,10]$.

Клинические данные, подтверждающие роль МСД в развитии ДДФ у больных с неизмененными коронарными артериями, базируются на ограниченном числе работ. Так, в одном из довольно обширных исследований ретроспективно оценили 376 историй болезни пациентов с ФВЛЖ $\geq 50 \%$ и без признаков обструктивного поражения коронарных сосудов [11]. У всех пациентов с СН с сохранной ФВЛЖ и признаками ДДФ было выявлено снижение коронарного резерва по данным ПЭТ. По мнению авторов работы, это может указывать на связь между микрососудистыми нарушениями, ДДФ и СН. Еще одной известной причиной развития ДДФ у пациентов с H/MKA считают гипертрофию миокарда (ГМ). Так, при оценке диастолической функции с помощью трансэзофагеальной допплерографии у 30 пациентов с ГМЛЖ 16 пациентов имели признаки ишемии и неизмененные КА, 14 - бессимптомную ГМЛЖ. Признаки ДДФ были обнаружены у пациентов обеих групп, но более выраженными оказались у пациентов группы 1 [12].

K возможным факторам риска ДДФ относят эктопическое отложение жира в миокарде, называемое стеатозом миокарда. В одном из исследований оценили содержание триглицеридов в миокарде и диастолическую функцию у 13 женщин с интактными КА с помощью протонной магнитно-резонансной спектроскопии и магнитно-резонансной маркировки тканей [6]. У 5 из них были выявлены признаки МСД и скрытой ДДФ, коррелирующие с более высоким содержанием триглицеридов в миокарде у этих пациенток. Авторы пришли к мнению, что стеатоз миокарда имеет четкую связь с развитием диастолической дисфункции у этой категории пациентов.

В то же время в мировой литературе имеются работы, указывающие на отсутствие ДДФ у данного контингента больных. Так, при оценке сердечной гемодинамики с помощью эхокардиографии у 99 пациентов с синдромом X, который был подтвержден нагрузочными пробами, не было обнаружено достоверных нарушений диастолической функции [13].
В связи с неоднозначными данными, представленными в литературе, важным и актуальным является продолжение исследований в этом направлении, определение критериев ДДФ у пациентов с Н/MКА с целью предотвращения развития диастолической сердечной недостаточности. В настоящее время наиболее доступным и распространенным методом оценки систолической и диастолической функций сердца остается ЭхоКГ. При всех достоинствах этот метод обладает недостаточной воспроизводимостью и значительно зависит от выполняющего оператора. Поэтому в клинической практике не меньшей популярностью пользуются радионуклидные методы, обладающие высокой точностью, воспроизводимостью, их результаты практически не зависят от выполняющего оператора.

Учитывая сказанное выше, целью данного исследования было оценить диастолическую функцию миокарда ЛЖ с помощью радионуклидного метода С-ОЭКТ/КТ миокарда у больных с болевым синдромом в области грудной клетки и $\mathrm{H} / \mathrm{MKA}$.

\section{ПАЦИЕНТЫ И МЕТОДЫ}

В исследование были включены 49 пациентов (24/25 мужчин/женщин). Критерии включения: возраст 43-77 лет (в среднем 62,6 \pm 8,9); наличие болевого синдрома (БС) в области грудной клетки, положительного результата нагрузочного теста, проведенного в амбулаторных условиях. Все пациенты проходили стационарное обследование и лечение на базе кафедры кардиологии РНИМУ им. Н. И. Пирогова.

Критерии исключения: в исследование не включали пациентов с АГ (систолическое артериальное давление $\geq 180$ и диастолическое артериальное давление $\geq 110$ мм рт. ст.), с поражением органов-мишеней и ГМЛЖ, гипертрофической и дилатационной кардиомиопатиями, перенесших острый инфаркт миокарда в течение ближайших шести месяцев, предшествующих исследованию, с ФВЛЖ < 45\%, постоянной формой трепетания и фибрилляции предсердий, нарушениями сердечного ритма и проводимости, заболеваниями клапанного аппарата, эндокринной, нервной системы, почечной и печеночной недостаточностью, иными заболеваниями, способствующими снижению резерва миокардиальной перфузии либо влияющими на систолическую и диастолическую функции миокарда.

Клиническое обследование включало сбор анамнеза, осмотр, регистрацию ЭКГ в 12 стандартных отведениях, холтеровское мониторирование ЭКГ, клинический и биохимический анализы крови, велоэргометрическую пробу (ВЭМ-проба), КАГ, а также С-ОЭКТ, совмещенную с рентгеновской КТ в покое и в сочетании с ВЭМ-пробой. По данным КАГ, у 37 обследованных КА были интактными, у 12 выявлены изменения только в одной КА со степенью стенозирования < 40\% и нормальная систолическая функция миокарда по данным ЭхоКГ. У 22 пациентов была обнаружена пограничная АГ или ГБ I стадии (индекс массы миокарда левого желудочка (ИММЛЖ) $132 \pm 21$ ) с достигнутыми целевыми значениями показателей АД (в среднем, 125/80 мм рт. ст.) на фоне медикаментозной терапии. У 17 пациентов иных сопутствующих заболеваний не выявлено (ИММЛЖ $128 \pm 27)$. У 21 пациента была выявлена гиперхолестеринемия: в целом по группе показатели общего холестерина (XC) составили 5,4 \pm 1,68 ммоль/л, ХС липопротеинов низкой плотности (ЛПНП) - 3,3 \pm 1,0 ммоль/л. У 10 обследованных заболевания сердечно-сосудистой системы были исключены, 
они составили контрольную группу. Клиническая характеристика больных представлена в табл. 1.

Всем больным с целью верификации ишемии миокарда и оценки систолической и диастолической функций сердца выполняли С-ОЭКТ/КТ миокарда в отделении радиоизотопной диагностики. Антиангинальную терапию отменяли перед проведением исследования на время, составляющее > 5 периодов полувыведения лекарственного средства.

С-ОЭКТ/КТ миокарда выполняли по двухдневному протоколу: в первый день проводили исследование в покое; во второй день - в сочетании с ВЭМ-пробой. ВЭМ-пробу выполняли под контролем ЭКГ и АД на велоэргометре фрирмы Ergoline по стандартному протоколу, согласно которому начальная нагрузка составляла 25 Вт с последующим увеличением на 25 Вт каждые 3 мин. При достижении критериев прекращения нагрузочной пробы внутривенно вводили радиофармпрепарат (РФП), после чего больной продолжал выполнять нагрузку в течение 1 мин.

В качестве РФП применяли отечественный Технеция (99mТс) сестамиби. РФП вводили внутривенно болюсом активностью 444-555 МБк. Регистрацию изображения на гамма-камере начинали через 60-90 мин при введении Технеция ( $\left.{ }^{99 \mathrm{~m}} \mathrm{Tc}\right)$ сестамиби в покое и через 45-60 мин при введении РФП во время проведения нагрузочной пробы.

ОЭКТ/КТ проводили в томографическом режиме, синхронизированном с зубцом R ЭКГ, на двухдетекторной гамма-камере «Symbia T-16» (Siemens; Германия), совмещенной с низкодозовым компьютерным томографом, оснащенным специальным коллиматором «SMARTZOOM» для проведения кардиологических исследований. Исследование выполняли в положении больного «лежа на спине» с вращением детекторов, расположенных под углом $90^{\circ}$ по отношению друг к другу, вокруг тела пациента на 180. Регистрировали 32 проекции, по 16 проекций каждым детектором.

C помощью программы QPS/QGS, AutoSPECT (Quark Inc., версия 8.5) оценивали количественные показатели перфузии и диастолической функции (ДФ) миокарда по 17-сегментарной модели ЛЖ. Оценивали следующие показатели систолической и диастолической функций:

1. Показатели систолической функции:

- ФВ $\geq 50 \%$ в состоянии покоя и после нагрузочной пробы;

- PER (ПСИ, КСО/с, в норме 2-3) - пиковая объемная скорость изгнания ЛЖ в покое и после нагрузочной пробы.

2. Показатели диастолической функции:

- PFR (ПСН, КДО/с, в норме 2-3) - пиковая объемная скорость наполнения ЛЖ, характеризует диастолическую функцию в целом;

- MFR/3 (ССН за 1/3, КДО/с, в норме 1,5-2) - средняя скорость наполнения ЛЖ в первую треть диастолы;

- PFR2 (ПСН2, в норме отсутствует) - при наличии в диастоле двух и более пиков наполнения ЛЖ вычисляли пиковую объемную скорость наполнения желудочка во время 2-го пика;

- TTPF (в норме 100-150мс) - время от начала диастолы до пикового уровня наполнения ЛЖ.

Отклонение хотя бы одного из показателей ДФ от нормальных значений расценивали как ДДФ [10].

Статистическую обработку данных проводили с использованием пакета статистических программ Statistica 6.0 (StatSoft; версия 6.0) Данные представлены в виде $\mathrm{M} \pm \mathrm{SD}$ (средняя величина \pm стандартное отклонение). Для сравнения качественных переменных использовали хи-квадрат Пирсона. При количестве наблюдений в одной из сравниваемых групп менее 11 применяли хи-квадрат Пирсона с поправкой Йетса. А при количестве наблюдений менее 5 - двусторонний точный критерий Фишера.

Для сравнения количественных независимых переменных применяли методы непараметрической статистики: для двух переменных - критерий Манна-Уитни, трех и более - Краскела-Уоллиса.

\section{РЕЗУЛЬТАТЫ ИССЛЕДОВАНИЯ}

По результатам обследования было ссормировано три группы. Группу 1 составили 17 пациентов с микрососудистой стенокардией (МСС): наличие болевого синдрома в грудной клетке, отсутствие сопутствующих заболеваний, способствующих снижению резерва миокардиальной перфузии, интактные коронарные артерии по данным коронароангиографии и верисицированная преходящая ишемия миокарда по данным C-ОЭКТ/КT. В группу 2 вошли 22 пациента с пограничной АГ, или ГБ І стадии, подтвержденной результатами суточного мониторирования АД, без ГМЛЖ (ИММЛЖ $132 \pm 21)$, без поражения органов-мишеней, с достигнутыми целевыми значениями АД и верифицированной преходящей ишемией миокарда, по данным С-ОЭКТ/КТ, ассоциирующейся с вторичной МСД. Группу 3 составили 10 практически здоровых лиц, у которых были исключены заболевания сердечнососудистой системы, изменения коронарных артерий при КАГ и нарушения перфузии миокарда при проведении ОЭКТ (группа сравнения). Пациенты во всех группах были сопоставимы по возрасту, индексу массы тела (ИМТ), частоте сердечных сокращений (ЧСС), АД, измеренных в состоянии покоя. В целом по группам не было достоверных различий по таким гемодинамическим параметрам, как ЧСС, АД, КСР, КДР (по данным эхокардиографии), измеренным в состоянии покоя (см. табл. 1).

Следует отметить, что у обследованных лиц контрольной группы показатели систолической и диастолической функций миокарда ЛЖ были в пределах нормальных значений и в покое, и после пробы с физической нагрузкой.

У обследованных больных с Н/MKA не было выявлено достоверных различий между показателями, характеризующими систолическую функцию миокарда ЛЖ. ФВ и пиковая объемная скорость изгнания крови из желудочков в покое и после пробы с физической нагрузкой на велоэргометре достоверно не различались у больных групп 1 и 2: ФВ в покое составила 58,5 \pm 6,6 и 59,5 \pm 6,8 (p > 0,05) соответственно, после нагрузки - 65,6 \pm 7,8 и 66,4 \pm 8,8 ( $p>0,05)$ соответственно. PER у пациентов группы 1 составила в покое 2,5 $\pm 0,2$, после нагрузки $2,7 \pm 0,3 ;$ у пациентов группы $2-2,4 \pm 0,3$ и 2,7 \pm 0,4 ( $p>0,05)$ соответственно.

Локальных нарушений систолической функции миокарда ЛЖ у обследованных больных обеих групп не было выявлено.

Нарушения со стороны ДФ миокарда ЛЖ в покое и после ВЭМ-пробы были выявлены у большинства обследованных больных с Н/MKA. У всех пациентов группы 1 и 17 пациентов группы 2 выявляли изменения хотя бы одного параметра, свидетельствующие о ДДФ. Обращает на себя внимание изменение практически во всех случаях показателя MFR/3. Однако чаще выявляли сочетание изменений нескольких показателей. Так, в покое в группе 1 у 5 пациентов выявлено изменение только 
одного параметра (MFR/3), у 6 пациентов - сочетание изменений двух параметров (при этом один из них MFR/3), у 5 пациентов были изменены три параметра и у одного пациента - все 4 параметра. При нагрузке отклонение от нормальных значений только одного параметра выявлялось у 3 пациентов, двух - у 4 пациентов, трех - у 7 и четырех - у 2 пациентов. Как видно из представленных данных, после нагрузочной пробы изменение трех и четырех показателей, характеризующих ДФ, отмечено у большего числа пациентов по сравнению с исследованием в покое.

В группе 2 в покое нарушение только одного показателя обнаружено у 2 пациентов, двух - у 7, трех - у 5 и четырех - у 3 пациентов. В то же время у 5 пациентов не выявлено изменений ни одного параметра. В ответ на нагрузку изменения только одного показателя определялись лишь у 1 пациента, двух - у 7, трех - у 6 и четырех - у 4 пациентов.

Средние значения показателей, характеризующих ДФ у больных групп 1 и 2 с Н/MKA и в контрольной группе, представлены в табл. 2.

Между пациентами групп 1 и 2 выявлена достоверная разница при сравнении PFRrest, MFR/3stress, TTPFrest, PFR2stress. Количество выявленных двух пиков наполнения ЛЖ в покое отмечалось у 3 (17\%) пациентов в группе 1 и 5 (23\%) - в группе 2; после нагрузки - у 4 (23\%) и 7 (32\%) соответственно. Достоверной разницы MFR/3 в покое и после нагрузки между пациентами групп 1 и 2 не получено. Возможно, это связано с тем, что нарушения этого параметра уже есть в обеих группах и в покое, и в нагрузке.

При сравнении с группой контроля выявлена достоверная разница в группе 1 во всех случаях, кроме

Таблица 1. Клинические показатели исследуемых групп пациентов

\begin{tabular}{|c|c|c|c|}
\hline Показатели & Группа 1 (МСС), $n=17$ & Группа 2 (МСД), $n=22$ & Группа 3 сравнения, $n=10$ \\
\hline Возраст & $62,7 \pm 7,8(50-77)$ & $60,6 \pm 8,1(44-75)$ & $63,2(43-74)$ \\
\hline Пол, м/ж & $6 / 11$ & $12 / 10$ & $6 / 4$ \\
\hline ИМТ (единицы измерения) & $25,8 \pm 3,6$ & $26,7 \pm 3,2$ & $25,7 \pm 3,47$ \\
\hline Длительность заболевания, годы & $3,4 \pm 2$ & $3,8 \pm 1,9$ & $3,2 \pm 1,7$ \\
\hline Систолическое АД (мм рт. ст.) & $122 \pm 9,2$ & $130 \pm 6,8$ & $126 \pm 6,4$ \\
\hline Диастолическое АД (мм рт. ст.) & $73 \pm 5,8$ & $74 \pm 4,0$ & $72 \pm 6,0$ \\
\hline иммЛЖ & $128 \pm 27$ & $132 \pm 21$ & $127 \pm 18$ \\
\hline ЧСС в покое & $67 \pm 8$ & $72 \pm 6$ & $66 \pm 8$ \\
\hline \multicolumn{4}{|l|}{ Характеристика боли } \\
\hline $\begin{array}{l}\text { Неангинозная (локальная, от нескольких часов и более, } \\
\text { нитроглицерином не купируется) }\end{array}$ & 5 & 7 & 10 \\
\hline Типичная ангинозная & 12 & 15 & 0 \\
\hline \multicolumn{4}{|l|}{ Данные ХМ-ЭКГ: } \\
\hline Норма & 6 & 11 & 10 \\
\hline Депрессия ST> 2,0 мм & 4 & 5 & 0 \\
\hline Депрессия ST<2,0 & 7 & 6 & 0 \\
\hline \multicolumn{4}{|l|}{ Данные эхокардиографии } \\
\hline ФВ ЛЖ (по методу Симпсона), \% & $64,5 \pm 9,5$ & $60,7 \pm 4,0$ & $67,4 \pm 10,2$ \\
\hline $\mathrm{KCP}, \mathrm{CM}$ & $3,5 \pm 0,3$ & $3,6 \pm 0,3$ & $2,77 \pm 0,5$ \\
\hline КДР, см & $4,8 \pm 0,3$ & $4,9 \pm 0,4$ & $4,65 \pm 0,2$ \\
\hline T3C, cM & $1,0 \pm 0,06$ & $1,04 \pm 0,08$ & $0,98 \pm 0,15$ \\
\hline
\end{tabular}

Примечание: МСС — микрососудистая стенокардия; КСР — конечный систолический размер; КДР — конечный диастолический размер; ТЗС — толщина задней стенки; ЧСС - частота сердечных сокращений.

Таблица 2. Количественные показатели диастолической функции в исследуемых группах пациентов

\begin{tabular}{|c|c|c|c|c|c|c|c|c|}
\hline \multirow{3}{*}{ Показатели } & \multicolumn{4}{|c|}{ Показатели ДФ } & \multicolumn{4}{|c|}{ p- различие между группами } \\
\hline & $\begin{array}{c}\text { Группы } 1 \text { и } 2 \\
n=39\end{array}$ & $\begin{array}{c}\text { Группа } 1 \\
n=17\end{array}$ & $\begin{array}{c}\text { Группа } 2 \\
n=22\end{array}$ & $\begin{array}{c}\text { Группа } 3 \text { (контроль) } \\
n=10\end{array}$ & \multirow{2}{*}{$\begin{array}{c}1 \text { и } 4 \\
\text { столбцы }\end{array}$} & \multirow{2}{*}{$\begin{array}{c}2 \text { и } 3 \\
\text { столбцы }\end{array}$} & \multirow{2}{*}{$\begin{array}{c}2 \text { и } 4 \\
\text { столбцы }\end{array}$} & \multirow{2}{*}{$\begin{array}{c}3 \text { и } 4 \\
\text { столбцы }\end{array}$} \\
\hline & 1 & 2 & 3 & 4 & & & & \\
\hline PFRstress, КДО/с & $1,89 \pm 0,21$ & $1,93 \pm 0,17$ & $1,86 \pm 0,24$ & $2,75 \pm 0,31$ & 0,000001 & 0,5 & 0,00002 & 0,000009 \\
\hline PFRrest, КДО/с & $1,97 \pm 0,27$ & $2,12 \pm 0,28$ & $1,86 \pm 0,20$ & $2,32 \pm 0,32$ & 0,002 & 0,003 & 0,14 & 0,0001 \\
\hline MFR/3 stress, КДО/с & $1,12 \pm 0,22$ & $1,01 \pm 0,23$ & $1,20 \pm 0,17$ & $1,77 \pm 0,14$ & 0,000001 & 0,009 & 0,00002 & 0,000009 \\
\hline MFR/3rest, КДО/с & $1,26 \pm 0,24$ & $1,26 \pm 0,24$ & $1,26 \pm 0,25$ & $1,60 \pm 0,15$ & 0,0003 & 0,9 & 0,0008 & 0,001 \\
\hline TTPFrest, mc & $164,1 \pm 18,1$ & $156,0 \pm 13,1$ & $170,4 \pm 19,2$ & $138,4 \pm 9,5$ & 0,000047 & 0,015 & 0,001 & 0,00005 \\
\hline TTPFstress, Mc & $172,3 \pm 24,9$ & $166,3 \pm 13,5$ & $176,9 \pm 30,5$ & $153,0 \pm 6,8$ & 0,01 & 0,3 & 0,009 & 0,04 \\
\hline $\begin{array}{l}\text { PFR2stress, число пациентов, } \\
\text { абс. число, \% }\end{array}$ & $14(35,9 \%)$ & $3(17,6 \%)$ & $11(50,0 \%)$ & 0 & - & 0,049 & - & - \\
\hline $\begin{array}{l}\text { PFR2rest, число пациентов, } \\
\text { абс. число, \% }\end{array}$ & $8(20,5 \%)$ & $1(5,9 \%)$ & 7 (31,8\%) & 0 & - & 0,1 & - & - \\
\hline
\end{tabular}

Примечание: значимые различия выделены жирным шрифтом; stress — показатель после нагрузки; rest — показатель в состоянии покоя. 
PFRrest, в группе 2 - во всех случаях сравнения. Следует отметить, что по данным ЭхоКГ признаки ДДФ выявляли у 11 (65\%) пациентов группы 1 и 14 (82\%) пациентов группы 2. К эхокардиографическим критериям ДДФ ЛЖ относят: снижение скорости раннего диастолического пика (е) (е` средний < 9 cм/c), увеличение соотношения между пиками трансмитрального кровотока (> 15), а также комбинацию некоторых показателей [12]. У всех пациентов, у которых по данным ЭхоКГ регистрировали ДДФ, определяли нарушения ДФ по данным С-ОЭКТ.

В качестве клинического примера приводим выписку из истории болезни пациентки Ш., 69 лет, с болевым синдромом в области грудной клетки при неизмененных коронарных артериях (стенокардия напряжения II ФК). Боли за грудиной и чувство нехватки воздуха длительностью до 25 мин возникали при эмоциональной нагрузке, нитроглицерином полностью не купировались. Анамнез: боли беспокоят около 2 лет, возникают практически ежедневно. Семейный анамнез не отягощен. Вредные привычки отрицает.

При поступлении состояние удовлетворительное ИМТ 20. Показатели клинического, биохимического анализов крови, а также уровни гормонов щитовидной железы в пределах нормы. ЭКГ: ритм синусовый правильный, ЧСС 68 в мин. ЭхоКГ: КДР 4,6 см, КСР 3,2 см, ТЗС 1,0см. ФВ > 60\%, нарушений локальной сократимости нет. При проведении ВЭМ-пробы в амбулаторных условиях накануне госпитализации выявлены эпизоды депрессии сегмента ST до 1,5 мм. По результатам КАГ коронарные сосуды интактны.

По данным С-ОЭКТ (рис. 1, 2), выполненной в покое (1) и после ВЭМ-пробы (2), показатели систолической функции миокарда ЛЖ не изменены: ФВЛЖ составила $67 \%$ в покое и 76\% после пробы с физической нагрузкой; локальные нарушения движения стенок ЛЖ не регистрируются; PER ЛЖ составила 2,7 в покое и 2,94 при нагрузке.

PFR ЛЖ в пределах нормы: в покое - 2,71, после ВЭМ-пробы - 3,04; MFR/3 ЛЖ изменена и составляет в покое 0,77, после нагрузки - 0,91. TTPF ЛЖ нарушено и составляет 286 в покое и 287 после нагрузки. Дополнительные пики наполнения ЛЖ не выявлены.

У пациентки обнаружены признаки ДДФ, о чем свидетельствуют измененные показатели MFR/3 и TTPF. По данным перфузионной ОЭКТ миокарда, зарегистрированы признаки преходящей ишемии миокарда в области верхушки и медиальных областях передней стенки.

\section{ОБСУЖДЕНИЕ РЕЗУЛЬТАТОВ}

В настоящем исследовании ДДФ выявлена у большинства обследованных больных с Н/МКА. Какие факторы могут способствовать нарушению процессов диастолы у данной категории больных? В качестве одной из причин ДДФ в литературе рассматривают фиброзные изменения миокарда, сопровождающиеся нарушениями локальной сократимости [3, 6]. В настоящем исследовании у всех пациентов систолическая функция миокарда ЛЖ была не нарушена, ФВЛЖ и PER из желудочков в покое и после пробы с физической нагрузкой находились в пределах нормальных значений, локальные нарушения движения стенок ЛЖ не определялись. Следовательно, в данном исследовании выявленные нарушения ДФ у пациентов обеих групп, по всей вероятности, не ассоциируются с нарушениями локальной сократимости (с фиброзными изменениями миокарда).

В качестве еще одной из причин ДДФ обсуждается гипертрофия миокарда ЛЖ. В данное исследование пациентов с ГМЛЖ не включали, у всех пациентов ИММЛЖ оставался в пределах нормы и составил $128 \pm 27$ и $132 \pm 21$ в группах 1 и 2 соответственно, и $127 \pm 18$ в группе контроля.

Что касается метаболических сдвигов как фактора возникновения ДДФ, то в исследование не вошли пациенты с заболеваниями, которые могли бы с таковыми ассоциироваться, если не принимать во внимание метаболические сдвиги, сопровождающие развитие преходящей ишемии миокарда.

В качестве наиболее вероятной основы для возникновения ДДФ у обследованной категории пациентов

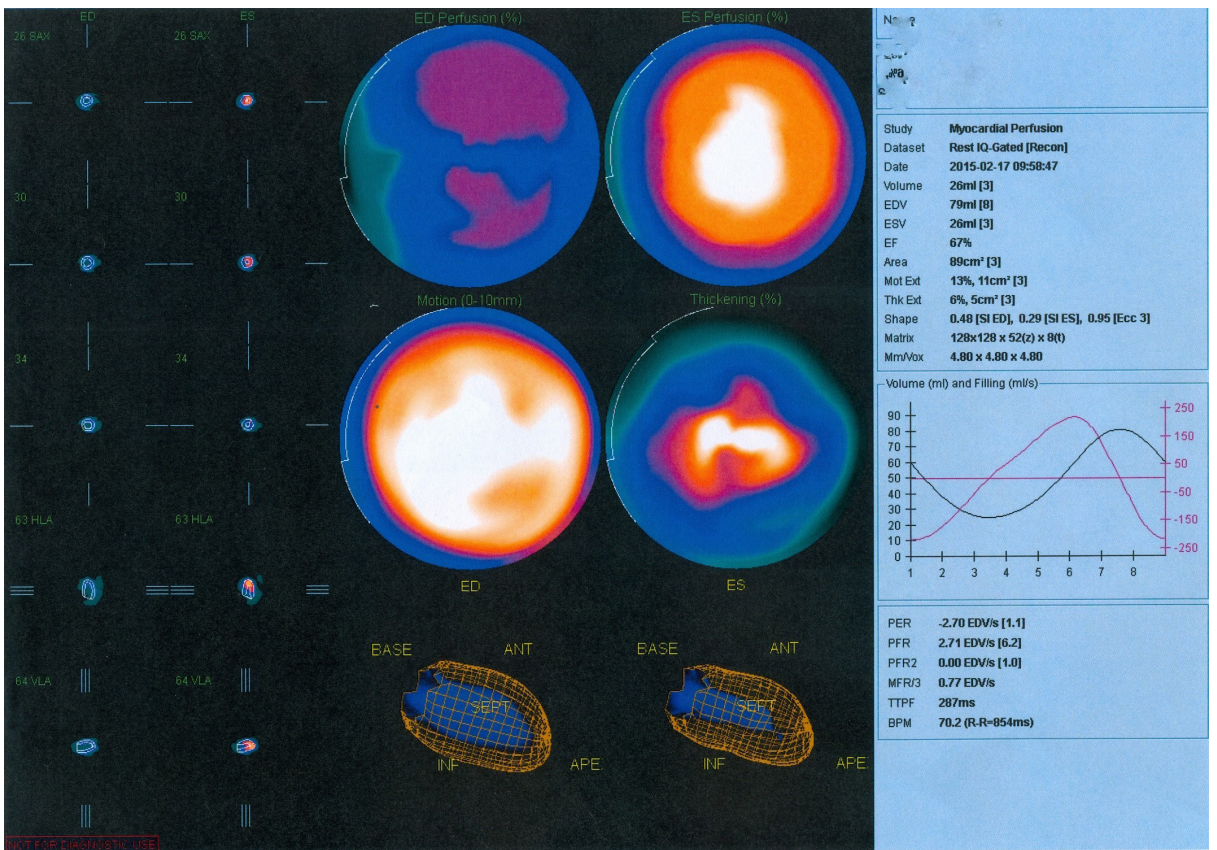

Рис. 1. С-ОЭКТ пациентки Ш., 69 лет, с болевым синдромом в области грудной клетки при неизмененных коронарных артериях (стенокардия напряжения ॥ ФК) в покое 


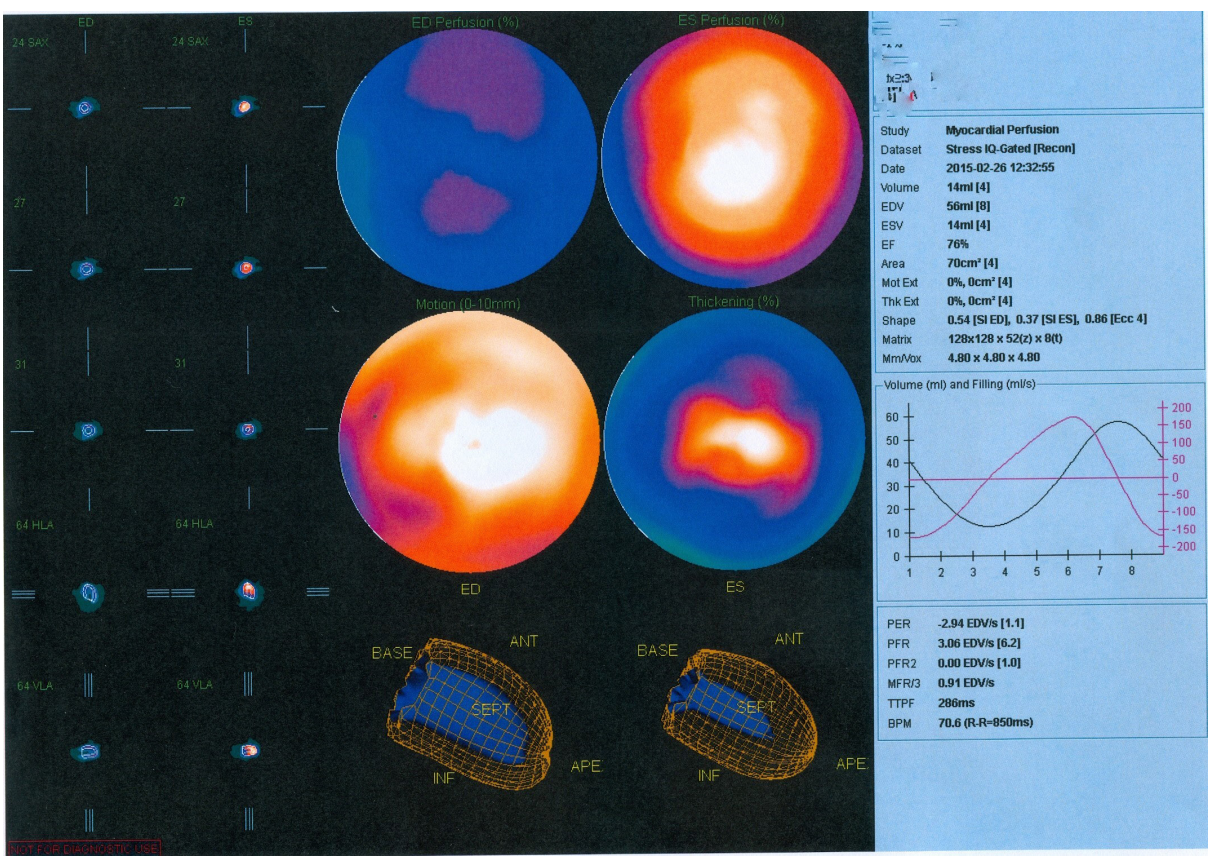

Рис. 2. С-ОЭКТ пациентки Ш., 69 лет, с болевым синдромом в области грудной клетки при неизмененных коронарных артериях (стенокардия напряжения ॥ ФК) после нагрузки

можно обсудить микрососудистую диссункцию. Коронарные артерии у больных в данном исследовании не изменены, но по данным ОЭКТ/КТ у пациентов и группы 1, и группы 2 верифицирована преходящая ишемия миокарда.

У больных группы 2 показатели PFR ЛЖ и ТTPF ЛЖ были изменены в большей степени, чем у больных группы 1 без сопутствующей ГБ. MFR/3, наиболее чувствительный параметр диастолической функции, была изменена уже в покое, по сравнению с группой контроля, но достоверно не различалась между группами 1 и 2. В ответ на нагрузку при отсутствии локальных нарушений движения стенок ЛЖ изменения MFR/3 стали более значимыми. Вероятно, причиной этому явилось снижение резерва миокардиального кровотока и преходящая ишемия миокарда.

Помимо этого, у части пациентов обеих групп было обнаружено появление 2-го пика наполнения ЛЖ в покое и увеличение количества этих пиков в ответ на нагрузку.

Полученные в настоящем исследовании данные согласуются с результатами других исследований, посвященных диастолической дисфункции у пациентов с неизмененными КА. Так, нарушения диастолической функции у пациенток с МBC продемонстрированы с использованием метода допплер-эхокардиографии [14]. В другом исследовании оценивали ДФ параллельно методами С-ОЭКТ, ЭхоКГ и МРТ сердца [15]. Данные
С-ОЭКТ полностью согласуются с результатами настоящего исследования. Что касается оценки показателей диастолической функции с помощью ЭхоКГ и С-ОЭКТ в работе указанных авторов, то по данным ЭхоКГ ДДФ выявлена более чем у 60\% пациентов с ДДФ, выявленной по данным С-ОЭКТ, что также совпадает с результатами настоящей работы (65 и 82\% в группах 1 и 2 соответственно). Таким образом, по мнению Y.W. Wu с соавторами, С-ОЭКТ оказался более чувствительным в выявлении ДДФ в сравнении с допплер-эхокардиографией. В то же время авторы отмечают большую чувствительность 64-срезового КТ в оценке ДФ [15].

Следует отметить, что все перечисленные исследования, включая настоящее, проведены на относительно небольшом количестве пациентов, имеют значимые ограничения. В связи с этим данная тема нуждается в дальнейшем изучении.

\section{ВЫВОДЫ}

Настоящее исследование продемонстрировало наличие некоторых нарушений диастолической функции у пациентов с неизмененными КА и болевым синдромом в грудной клетке. В связи с тем, что по данному вопросу в литературе встречаются единичные и в некоторой степени противоречивые сведения, тема нуждается в дальнейшем изучении.

\section{Литература}

1. Hogg K, Swedberg K, McMurray J. Heart Failure with preserved left ventricular systolic function. Epidemiology, Clinical Characteristics, and Prognosis. J Am Coll Cardiol. 2004; 43 (3): 317-27.

2. Фомин И. В., Беленков Ю. Н., Мареев В. Ю. и др. Распространенность хронической сердечной недостаточности в Европейской части РФ (данные исследования «ЭПОХА $\mathrm{XCH}$ ». ЖKCH, 2006; 3 (7): 112-15.

3. Paulus WJ, Tschope C. A novel paradigm for heart failure with

preserved ejection fraction: comorbidities drive myocardial dysfunction and remodeling through coronary microvascular endothelial inflammation. J Am Coll Cardiol. 2013; 62: 263-71.

4. Taqueti VR, Ridker PM. Inflammation, coronary flow reserve, and microvascular dysfunction: moving beyond cardiac syndrome $X$. JACC Cardiovasc Imaging. 2013; (6): 668-71.

5. Crea F, Merz CNB, Beltrame JF, et al. Coronary Vasomotion Disorders International Study Group. The parallel tales of microvascular angina and heart failure with preserved ejection 
fraction: a paradigm shift. Eur Heart J. 2017; (38): 473-77.

6. Wei J, Nelson MD, Szczepaniak EW, et al. Myocardial steatosis as a possible mechanistic link between diastolic dysfunction and coronary microvascular dysfunction in women Am J Physiol Heart Circ Physiol. 2016; 310 (1): H14-H1.

7. Ponikowski P, Voors AA, Anker SD, et al. 2016 ESC Guidelines for the diagnosis and treatment of acute and chronic heart failure: the task force for the diagnosis and treatment of acute and chronic heart failure of the European Society of Cardiology (ESC) developed with the special contribution of the Heart Failure Association (HFA) of the ESC. Eur Heart J. 2016; (37): 2129-200.

8. Redfield MM. Heart failure with preserved ejection fraction. N Engl J Med. 2016; (375): 1868-77.

9. Van Krajj DJ, van Pol PE, Ruiters AW, et al. Diagnosing diastolic heart failure. Eur J Heart Fail. 2002; 4 (4): 419-30.

10. Kato S, Saito N, Kirigaya $\mathrm{H}$, et al. Impairment of coronary flow reserve evaluated by phase contrast cine-magnetic resonance imaging in patients with heart failure with preserved ejection fraction. J Am Heart Assoc. 2016; (5): e002649.

11. Srivaratharajah K, Coutinho T, Liu P, et al. Reduced myocardial flow in heart failure patients with preserved ejection fraction. Circ Heart Fail. 2016; 9 (7): e002562.

12. Isaaz K, Bruntz JF, Paris D, et al. Abnormal coronary flow velocity pattern in patients with left ventricular hypertrophy, angina pectoris, and normal coronary arteries: A transesophageal Doppler echocardiographic study. American Heart Journal. 1994; 128 (3): 500-10.

13. Kaski JC, Rosano GMC, Collins $P$, et al. Cardiac syndrome $X$ : clinical characteristics and left ventricular function. Journal of the American College of Cardiology. 1995; (25): 807-14.

14. Nelson MD, Szczepaniak LS, Weiet J, al. Diastolic Dysfunction in Women With Signs and Symptoms of Ischemia in the Absence of Obstructive Coronary Artery Disease A Hypothesis-Generating Study. Circ Cardiovasc Imaging. 2014; (7): 510-6.

15. Wu Y, Tadamura E, Yamamuro M, et al. Estimation of global and regional cardiac function using 64-slice computed tomography: a comparison study with echocardiography, gated-SPECT and cardiovascular magnetic resonance. Int J Cardiol. 2008; 128 (1): 69-76.

\section{References}

1. Hogg K, Swedberg K, McMurray J. Heart Failure with preserved left ventricular systolic function. Epidemiology, Clinical Characteristics, and Prognosis. J Am Coll Cardiol. 2004; 43 (3): 317-27.

2. Fomin IV, Belenkov JuN, Mareev Vju, et al. Rasprostranennost hronicheskoj serdechnoj nedostatochnosti v Evropejskoj chasti RF (dannye issledovanija "JePOHA - HSN"). ZhSN. 2006; 3 (7): 112-5.

3. Paulus WJ, Tschope C. A novel paradigm for heart failure with preserved ejection fraction: comorbidities drive myocardial dysfunction and remodeling through coronary microvascular endothelial inflammation. J Am Coll Cardiol. 2013; 62: 263-71.

4. Taqueti VR, Ridker PM. Inflammation, coronary flow reserve, and microvascular dysfunction: moving beyond cardiac syndrome $\mathrm{X}$. JACC Cardiovasc Imaging. 2013; (6): 668-71.

5. Crea F, Merz CNB, Beltrame JF, et al. Coronary Vasomotion Disorders International Study Group. The parallel tales of microvascular angina and heart failure with preserved ejection fraction: a paradigm shift. Eur Heart J. 2017; (38): 473-77.

6. Wei J, Nelson MD, Szczepaniak EW, et al. Myocardial steatosis as a possible mechanistic link between diastolic dysfunction and coronary microvascular dysfunction in women Am J Physiol Heart Circ Physiol. 2016; 310 (1): H14-H1.

7. Ponikowski P, Voors AA, Anker SD, et al. 2016 ESC Guidelines for the diagnosis and treatment of acute and chronic heart failure: the task force for the diagnosis and treatment of acute and chronic heart failure of the European Society of Cardiology (ESC) developed with the special contribution of the Heart Failure Association (HFA) of the ESC. Eur Heart J. 2016; (37): 2129-200.

8. Redfield MM. Heart failure with preserved ejection fraction. N Engl J Med. 2016; (375): 1868-77.

9. Van Krajj DJ, van Pol PE, Ruiters AW, et al. Diagnosing diastolic heart failure. Eur J Heart Fail. 2002; 4 (4): 419-30.

10. Kato S, Saito N, Kirigaya H, et al. Impairment of coronary flow reserve evaluated by phase contrast cine-magnetic resonance imaging in patients with heart failure with preserved ejection fraction. J Am Heart Assoc. 2016; (5): e002649.

11. Srivaratharajah K, Coutinho T, Liu P, et al. Reduced myocardial flow in heart failure patients with preserved ejection fraction. Circ Heart Fail. 2016; 9 (7): e002562.

12. Isaaz K, Bruntz JF, Paris D, et al. Abnormal coronary flow velocity pattern in patients with left ventricular hypertrophy, angina pectoris, and normal coronary arteries: A transesophageal Doppler echocardiographic study. American Heart Journal. 1994; 128 (3): 500-10.

13. Kaski JC, Rosano GMC, Collins $P$, et al. Cardiac syndrome $X$ : clinical characteristics and left ventricular function. Journal of the American College of Cardiology. 1995; (25): 807-14.

14. Nelson MD, Szczepaniak LS, Weiet J, al. Diastolic Dysfunction in Women With Signs and Symptoms of Ischemia in the Absence of Obstructive Coronary Artery Disease A Hypothesis-Generating Study. Circ Cardiovasc Imaging. 2014; (7): 510-6.

15. Wu Y, Tadamura E, Yamamuro M, et al. Estimation of global and regional cardiac function using 64-slice computed tomography: a comparison study with echocardiography, gated-SPECT and cardiovascular magnetic resonance. Int J Cardiol. 2008; 128 (1): 69-76. 